\title{
Inelastic dark matter and DAMA/LIBRA: An experimentum crucis
}

\section{Citation}

Finkbeiner, Douglas P., Tongyan Lin, and Neal Weiner. 2009. "Inelastic Dark Matter and DAMA/ LIBRA: An Experimentum Crucis." Physical Review D 80 (11) (December 15). doi:10.1103/ physrevd.80.115008.

\section{Published Version}

10.1103/physrevd.80.115008

\section{Permanent link}

http://nrs.harvard.edu/urn-3:HUL.InstRepos:33461886

\section{Terms of Use}

This article was downloaded from Harvard University's DASH repository, and is made available under the terms and conditions applicable to Open Access Policy Articles, as set forth at http:// nrs.harvard.edu/urn-3:HUL.InstRepos:dash.current.terms-of-use\#OAP

\section{Share Your Story}

The Harvard community has made this article openly available.

Please share how this access benefits you. Submit a story.

Accessibility 


\title{
Inelastic Dark Matter and DAMA/LIBRA: An Experimentum Crucis
}

\author{
Douglas P. Finkbeiner, ${ }^{1}$ Tongyan Lin, ${ }^{2}$ and Neal Weiner ${ }^{3}$ \\ ${ }^{1}$ Harvard-Smithsonian Center for Astrophysics, 60 Garden St., Cambridge, MA 02138, USA \\ ${ }^{2}$ Physics Department, Harvard University, Cambridge, MA 02138, USA \\ ${ }^{3}$ Center for Cosmology and Particle Physics, Department of Physics, \\ New York University, New York, NY 10003, USA
}

(Dated: July 21, 2013)

\begin{abstract}
The DAMA/LIBRA collaboration has detected an annual modulation of the recoil rate in NaI crystals with the phase expected for WIMP scattering events. This signal is dramatically inconsistent with upper limits from other experiments for elastically scattering weak-scale WIMPs. However, the results are compatible for the case of inelastic dark matter (iDM). The iDM theory, as implemented by Tucker-Smith and Weiner, constrains the WIMP to a tight contour in $\sigma_{n}-\delta$ space, where $\delta$ is the mass difference between the ground state and excited WIMPs. An urgent priority in direct detection is to test this scenario. The crucial test of the iDM explanation of DAMA - an experimentum crucis - is an experiment with directional sensitivity, which can measure the daily modulation in direction. Because the contrast can be $100 \%$, it is a sharper test than the much smaller annual modulation in the rate. We estimate the significance of such an experiment as a function of the WIMP mass, cross section, background rate, and other parameters. The proposed experiment severely constrains the DAMA/iDM scenario even with modest exposure $(\sim 1000 \mathrm{~kg} \cdot$ day $)$ on gaseous xenon.
\end{abstract}

PACS numbers: 95.35.+d

\section{INTRODUCTION}

The DAMA claim [1] of an annual modulation signal has long appeared to be in conflict with non-detections in other experiments [2]. Though recent limits by XENON10 [3, 4] and CDMS II [5] appear to rule out the DAMA region of parameter space by a factor of 100 in cross section, DAMA/LIBRA [6] has recently confirmed their previous annual modulation result and increased the significance to $8.2 \sigma$. This conflict has motivated serious discussion of models beyond the simplest elastic scattering of weak-scale WIMPs, with the hope of accommodating DAMA as well as the other limits.

At least four approaches have been considered: 1. electron scattering [7]; 2. spin dependent scattering [8-10]; 3. light dark matter [11, 12]; and 4. inelastic scattering [13]. The first hypothesizes that the signal in DAMA is scattering of WIMPs off of electrons. Significant momentum can be transferred to the electron during the small fraction of the time $(<0.1 \%)$ that it finds itself near the nucleus and at moderately relativistic speeds. However, this small fraction must be balanced by an uncomfortably large cross section, which is almost certainly ruled out by early Universe (CMB) constraints.

The spin-dependent scattering argument attempts to circumvent limits from CDMS in Si for example by positing that the cross section is strongly dependent on nuclear spin. However, recent experiments [14] have significantly tightened constraints on this scenario, and the allowed regions require a significant drop in the background in the signal region [15]. While small regions of parameter space are still allowed, we do not consider this here.

Another suggestion is that the DAMA recoil events are not in the energy range first suspected. Assuming recoils off of iodine, the quenching factor of 0.09 implies that the $2-6$ keVee observed energy corresponds to a recoil energy of $22-66 \mathrm{keVr}$. It has recently been suggested that "channeling", i.e. alignment of the recoil with principal directions in the crystal lattice, creates an effective quenching factor of unity for some fraction of the events [16]. In this case, there is a small amount of parameter space available for lighter WIMPs $(\sim 5 \mathrm{GeV})$ still compatible with other limits [15, 17, 18]. In general, light WIMPs have difficulty with constraints from the energy spectrum of the unmodulated DAMA signal [19, 20]. While further exploration of light WIMPs may be warranted, we do not consider this option here.

\section{A. The DAMA/iDM Scenario}

The inelastic scattering scenario of Tucker-Smith \& Weiner [13, 21, 22] takes a different approach: inelastic dark matter (iDM) has an excited state some $\delta \sim 100 \mathrm{keV}$ above the ground state. The origin of this excited state is unimportant for the present arguments; see 23] for one realization of this idea. Elastic scatterings off of nuclei are suppressed by at least two orders of magnitude with respect to the inelastic scatterings, leading to a preferred energy threshold with few events at low energies. The high sensitivity of e.g. XENON10 to low-energy scatterings (which dominate in the standard elastic scattering models) means that even a small exposure time $(316 \mathrm{~kg}$ day) can place record-beating limits on the elastic cross section. Because iDM does not produce such low-energy events, it is plausible that the much larger combined exposure time of DAMA/LIBRA and DAMA/NaI (300,000 $\mathrm{kg}$ day) could see the higher energy events invisible in the other experiments.

Models of iDM are simple to construct, for in- 
stance a fourth-generation (vector-like) neutrino, coupling through the Z-boson [21], a mixed sneutrino [13], KK states in RS theories [24], in composite models 25], or in theories with light mediators [26], see also [24, 25, 27 36]. In fact, off-diagonal couplings are very natural in dark matter theories, with only the small splitting $\delta$ remaining to be explained.

In an annual modulation experiment, iDM enjoys an additional enhancement relative to elastic models because only WIMPs on the high velocity tail scatter. The modulation can be much larger than the $2-3 \%$ expected for elastic scattering, partially compensating for the fact that the majority of WIMPs are below threshold and do not scatter.

If the direct detection data from DAMA and others are taken at face value as nuclear WIMP scattering events, they argue strongly for further experiments designed to test iDM. The experiment must make predictions beyond the already observed annual modulation so that a positive result would add substantially to the believability of the result. Such a make-or-break experiment is known as a "critical experiment," or experimentum crucis [64]. In the next section we describe such an experiment and discuss the limits obtained.

\section{B. Advantages of Directional Sensitivity}

The DAMA result is compelling enough to motivate further experiments involving iodine or other nuclei of similar mass. Direct detection experiments generally fall into 3 categories, based on their background rejection strategy. Some (CDMS II, XENON10, etc.) reject individual electron scattering events and look for the residual signal from WIMP scattering.

Another strategy for dealing with background is to search for the annual modulation of the signal (DAMA) brought about by the Earth's velocity around the Sun, added to the velocity of the Sun around the Galaxy. The assumption is that the WIMP velocities are nearly isotropic, and the Sun moves through the WIMPs at roughly $200 \mathrm{~km} / \mathrm{s}$. The Earth moves around the Sun at $v_{\text {orb }} \approx 30 \mathrm{~km} / \mathrm{s}$ in an orbit inclined by $i \approx 60^{\circ}$ with respect to the Sun's velocity, introducing a modulation of $v_{\text {orb }} \cos (i) \approx 15 \mathrm{~km} / \mathrm{s}$. This method has the virtue of ignoring all steady state instrumental backgrounds, but is vulnerable to backgrounds that vary with the seasons. Though DAMA has placed stringent limits on variations in temperature, humidity, radon gas, line voltage, and anything else known to vary by season [6], this remains a persistent concern.

A third strategy is to use directional information 37. Because the scattering events should originate, on average, from a specific direction on the sky $\left(\ell=90^{\circ}, b=0^{\circ}\right)$, a daily modulation in direction due to the rotation of the Earth is a sharp test of the WIMP scattering model. As with the annual modulation, many other backgrounds may be expected to vary on a daily timescale. However, as the Earth orbits around the Sun, the angle between the Sun direction and the WIMP signal varies from $60^{\circ}(\sim 7$ March) to $120^{\circ}$ ( $\sim 9$ September). Also, any Sun-related oscillation $\left(365.25 \mathrm{yr}^{-1}\right)$ is orthogonal to the WIMP signal $\left(366.25 \mathrm{yr}^{-1}\right)$ over one year. This separation allows a much sharper test than the annual modulation alone, even in the limit of low statistics. Furthermore, directional detectors have excellent background rejection and can distinguish between recoils of nuclei and other particles by correlating the length and energy of recoil tracks.

In the context of iDM, a directional experiment has another advantage. The minimum velocity $v_{\min }$ for a WIMP to scatter with a nuclear recoil of energy $E_{R}$ is:

$$
v_{\text {min }}=\sqrt{\frac{1}{2 m_{N} E_{R}}}\left(\frac{m_{N} E_{R}}{\mu}+\delta\right)
$$

where $\mu$ is the nucleus-WIMP reduced mass $m_{\chi} m_{N} /\left(m_{\chi}+m_{N}\right)$ and $m_{\chi}$ is the WIMP mass. Because of the energy threshold, most events result from WIMPs in the high velocity tail of the WIMP velocity distribution, and therefore most events happen near threshold. This is advantageous because events at threshold have a sharply peaked angular distribution, making the directional discrimination even more pronounced. The energy-dependent maximum recoil angle is

$$
\cos \gamma_{\max }\left(E_{R}\right)=\frac{v_{e s c}-v_{\min }\left(E_{R}, \delta\right)}{v_{E}}
$$

Here $\gamma$ is the angle between the velocity of the Earth and the recoil velocity in the Earth frame, and $v_{e s c}$ is the Galactic escape velocity from the Solar neighborhood. For the benchmark models considered here, $\gamma$ is constrained to be within 100 degrees of the Earth's direction. Furthermore, as with annual modulation, the total number of events should vary through the year in a predictable way. These advantages allow a decisive test of the DAMA/iDM scenario with modest experimental effort.

In this article, we evaluate the sensitivities for the DAMA/iDM scenario as a function of WIMP mass $m_{\chi}$, $\delta$, and other parameters. We focus on a set of benchmark models, given in Table [ that can simultaneously explain DAMA and satisfy constraints from other experiments [22]. Note that the $m_{\chi}=70 \mathrm{GeV}$ benchmark cannot actually explain the DAMA data because of the predicted asymmetry in the modulation amplitude during summer and winter. However, we include the benchmark as a worst-case scenario, as there is flexibility in the WIMP parameters due to the uncertainty in the halo distribution and astrophysical parameters [38]. These benchmarks give the general features and sensitivities (within an order of magnitude) of a directional experiment to the available parameter space of iDM. We find that in most parts of parameter space, $1000 \mathrm{~kg}$ days of exposure is sufficient to confirm or refute DAMA/iDM at high confidence. 
a)
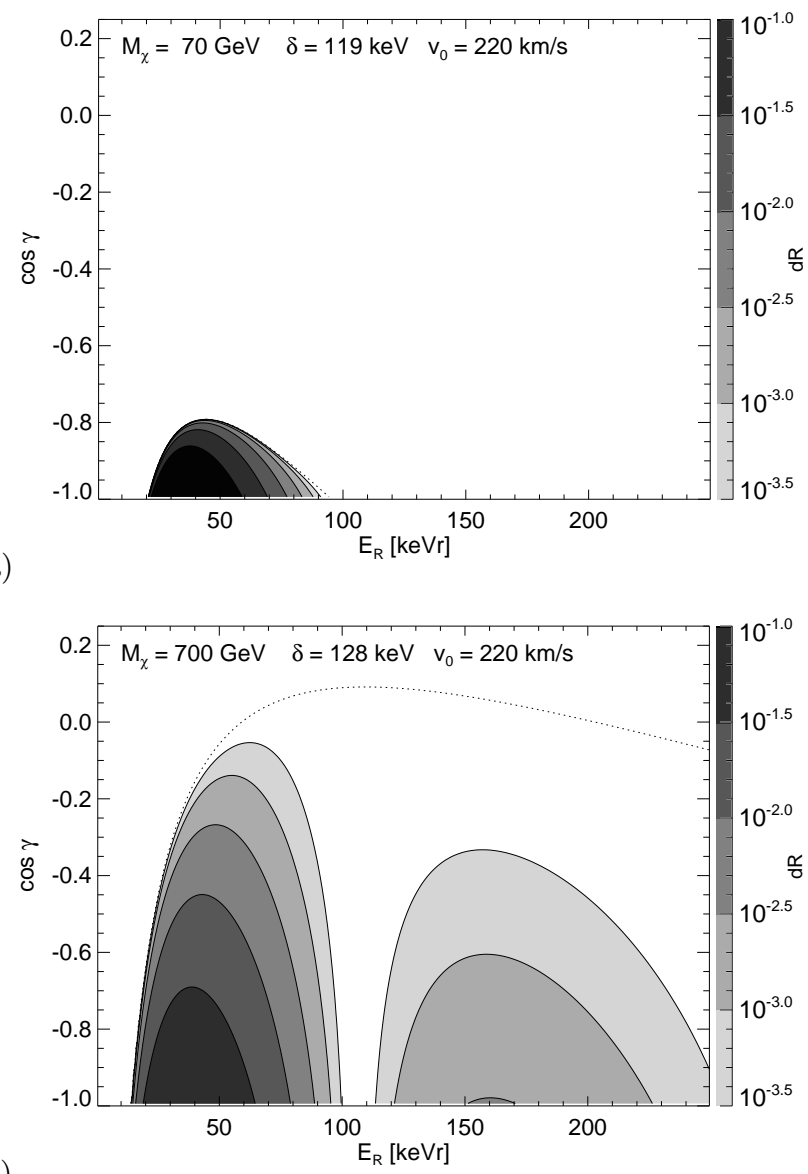

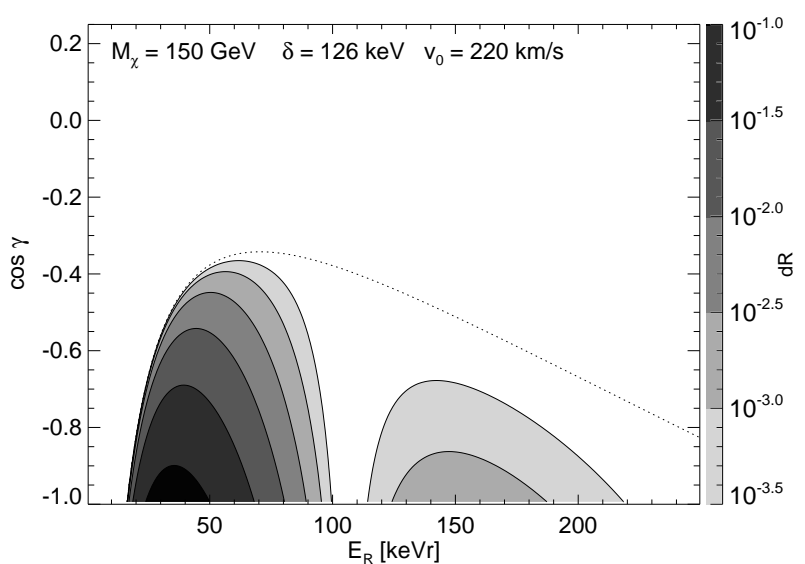

b)

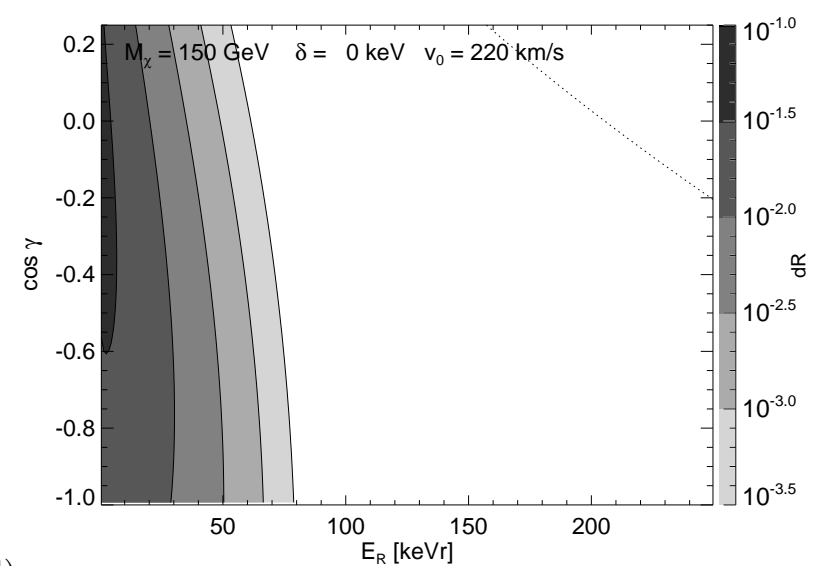

FIG. 1: Differential rates $d R /\left(d E_{R} d \cos \gamma\right)$ for the benchmark models given in Table $\Pi$ for $v_{e s c}=500 \mathrm{~km} / \mathrm{s}$, as well as for an elastic WIMP. In each case, the differential rate is normalized so that the total rate is unity. Outside the region indicated by the dashed line, scattering events are kinematically forbidden.

\begin{tabular}{ccc}
$\begin{array}{c}m_{\chi} \\
(\mathrm{GeV})\end{array}$ & $\begin{array}{c}\delta \\
(\mathrm{keV})\end{array}$ & $\begin{array}{c}\sigma_{n} \\
\left(10^{-40} \mathrm{~cm}^{2}\right)\end{array}$ \\
\hline 70 & 119 & 11.85 \\
150 & 126 & 2.92 \\
700 & 128 & 4.5 \\
$150^{*}$ & 130 & 4
\end{tabular}

TABLE I: Benchmark models for $v_{e s c}=500 \mathrm{~km} / \mathrm{s}, v_{0}=220$ $\mathrm{km} / \mathrm{s}$ [22]. In the last row we have listed the benchmark model for $m_{\chi}=150 \mathrm{GeV}$ at $v_{\text {esc }}=600 \mathrm{~km} / \mathrm{s}$.

\section{EXPERIMENTAL SETUP}

Before discussing the specifics of the experiment, we can address a few basic questions of exposure and energy range. DAMA/LIBRA reports a cumulative modulation in the $2-6 \mathrm{keVee}$ range of 0.052 counts per day per $\mathrm{kg}$, $(\mathrm{cpd} / \mathrm{kg})$. The quoted energy range is related to the nuclear recoil energy by a quenching factor $q=E_{e e} / E_{N R} \simeq$ 0.09 for iodine. Thus, $2-6 \mathrm{keVee} \approx 22-66 \mathrm{keVr}$.
In the extreme case where the modulation is $100 \%$ (i.e., no scattering at all occurs in the winter), the signal is essentially directional. One would need approximately $400 \mathrm{~kg}$ - day in the summer to yield 20 events of signal, roughly the number of events needed for an unambiguous detection at zero background, as we will discuss in Section IVA. Consistency with other experiments is also possible with $\sim 20 \%$ modulation [22], with only $40 \mathrm{~kg}$. day needed for a clear discovery.

However, this estimate assumes that the signal occurs in an energy range which is detectable at a directional experiment, and this, we shall see, is very unlikely to be the case. A directional experiment will likely have a higher energy threshold.

The DAMA/LIBRA signal peaks near $E_{R} \approx 3 \mathrm{keVee}$, after which it falls significantly. Above $5 \mathrm{keVee}$, the total modulation is $0.0034 \pm 0.0024 \mathrm{cpd} / \mathrm{kg}$, which is consistent with zero. The signal above 4 keVee yields a signal at DAMA of $0.014 \pm 0.004 \mathrm{cpd} / \mathrm{kg}$, which requires approximately $1400 \mathrm{~kg}$ · day of exposure for 20 events. Moreover, it is possible that the actual signal is at 3.5 keVee and 
below, and the signal at apparently higher energy is due to the resolution of the DAMA detector [39].

There is a significant uncertainty in the quenching factor as well. While $q=0.09$ is a commonly used value, the measurements are uncertain, and values $q=0.10$ and slightly higher are possible. Since the range of WIMP parameters allowed arises from fitting the DAMA peak, the uncertainty in this factor is hidden from our analyses here. Nonetheless, the presence of a larger quenching factor would result in a lower range of energies for the signal. Thus, it is clear that a robust test of the DAMA result involves pushing the energy threshold as low as possible. While the models that we consider generally do have signal above $50 \mathrm{keVr}$, this cannot be guaranteed, especially in situations where form factors might suppress the higher energy events [25]. In the event the experiments as we describe are performed and no signal is seen, we would advocate lowering the threshold, even at the cost of exposure from reduced pressure, to whatever extent possible.

With these important caveats in mind, we can proceed to discuss the details of what such an experiment would look like.

\section{A. Experimental Design}

Gaseous detectors can resolve the nuclear recoil tracks, which have lengths of several millimeters at sufficiently low pressures. Several gaseous directional detection experiments are already underway, including DMTPC [40], NEWAGE [41], DRIFT [42], and MIMAC [43], which employ time-projection chambers to reconstruct tracks. However, these experiments are typically focused on spindependent WIMP-nucleus interactions and use the gas $\mathrm{CF}_{4}$ as a detector, with the exception of DRIFT, which uses $\mathrm{CS}_{2}$. For a review of the various detector technolo- gies, see [44 46].

We suggest using a gas containing xenon or another heavy element. This increases sensitivity to spinindependent interactions because scattering rates are kinematically highly suppressed for lighter nuclei in the iDM scenario, in addition to the overall factor of $A^{2}$ that appears in the cross section. However, heavier elements have shorter recoil tracks which are more difficult to resolve. Furthermore, the gas should allow for good electron (or ion) drift and also have good scintillation properties (at least for DMTPC). Choosing a gas will involve some compromise between these properties. We note that for a splitting of $\delta \sim 120 \mathrm{keV}, A$ must be greater than 75 to see any signal for the mass range $m_{\chi} \sim 100-1000 \mathrm{GeV}$ for an earth velocity of $225 \mathrm{~km} / \mathrm{s}$ and an escape velocity of $500 \mathrm{~km} / \mathrm{s}$.

According to preliminary work of the directional detection experiments mentioned above, in order to resolve the angles of the tracks, the gas chamber must be at a pressure of around 50 torr. Furthermore if the recoil energies are too low (below $\sim 50 \mathrm{keVr}$ ), it is difficult to detect the sense (head-tail discrimination) of the track, which reduces sensitivity significantly [47-49]. The directional resolution of DMTPC is currently estimated to be around 15 degrees at $100 \mathrm{keVr}$ and improves by several degrees at higher energies [50].

The dominant irreducible background is neutron recoils arising from radioactive materials near or in the detector. Simulations suggest background rejection is excellent for gamma-rays, electrons, and $\alpha$ 's [51] (see also Fig. 7 of [40]). The DRIFT collaboration has reported on neutron backgrounds; however, they found a radioactive source $\left({ }^{222} \mathrm{Rn}\right)$ inside the detector [52]. The NEWAGE experiment at Kamioka estimated their primary background to come from the fast neutron flux which, when shielded by $50 \mathrm{~cm}$ of water, would contribute only a few events per year [53].

\section{RECOIL SPECTRUM}

We derive the differential nuclear recoil spectrum in recoil energy $E_{R}$ and $\cos \gamma$, which is defined as $\cos \gamma=\hat{v}_{E} \cdot \hat{v}_{R}$. The Earth's motion in the halo rest frame is $\vec{v}_{E}$ and the vector $\vec{v}_{R}$ is the nuclear recoil velocity in the Earth's frame. Let $\vec{v}$ be the incoming WIMP velocity in the Earth's frame.

The single nucleon scattering cross section is:

$$
d \sigma=\frac{\sigma_{n} m_{n}}{2 \mu_{n}^{2}} \frac{1}{v^{2}} d E_{R} d \cos \gamma \delta^{(1)}\left(\hat{v} \cdot \hat{v}_{R}-\frac{v_{m i n}}{v}\right)
$$

where $\mu_{n}$ is the WIMP-nucleon reduced mass and $\sigma_{n}$ is a reference cross section that is assumed to be the same for all nucleons. $m_{n}$ is nucleon mass. The minimum velocity $v_{\min }$ for a WIMP to scatter with a nuclear recoil of energy $E_{R}$ was given in Eq. 1]

The differential recoil rate for WIMP-nucleus scattering is

$$
\frac{d R}{d E_{R} d \cos \gamma}=N_{T} \frac{\rho_{\chi}}{m_{\chi}} \int d^{3} v v f\left(\vec{v}+\vec{v}_{E}\right) \frac{d \sigma}{d E_{R} d \cos \gamma}
$$

where $f(\vec{v})$, the WIMP distribution in the galaxy frame, is boosted to the Earth frame. $N_{T}$ is the number of target nuclei per $\mathrm{kg}$ and $\rho_{\chi}$ is the local WIMP energy density. We are now using the differential scattering cross section $d \sigma$ 
a)

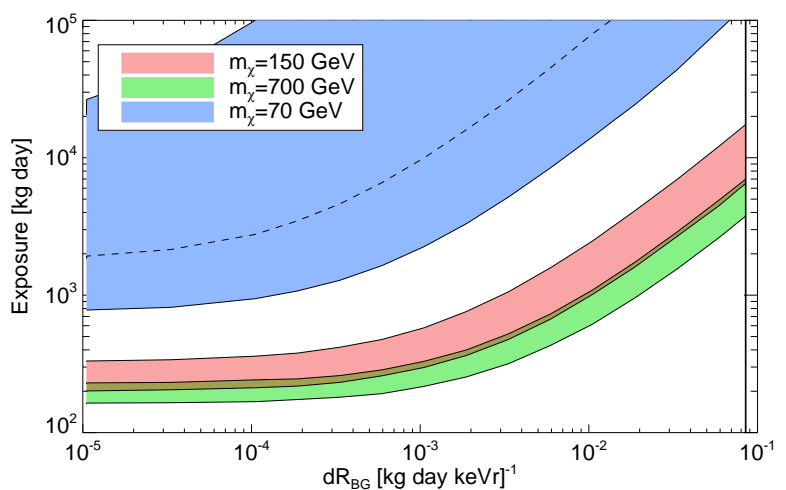

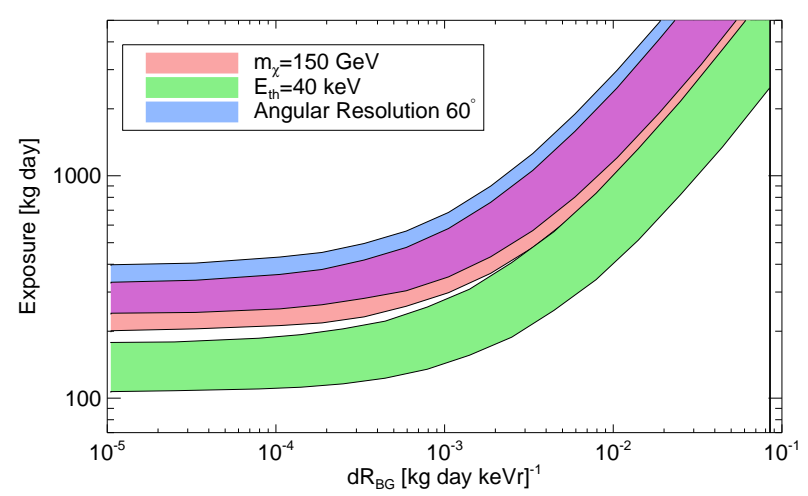

FIG. 2: Exposure to obtain a $5 \sigma$ measurement of $\langle\cos \gamma\rangle 90 \%$ of the time the experiment is conducted on Earth. The energy range of the experiment is $E_{R} \in[50,80] \mathrm{keVr} . d R_{B G}$ is the background rate; the DAMA unmodulated background rate is indicated by the solid vertical line at 0.085 . The bands shown give the exposures necessary as the rates modulate throughout a year. Since the annual modulation is asymmetric in summer and winter for low mass dark matter, the average exposure for $m_{\chi}=70 \mathrm{GeV}$ is indicated by the dashed line. In (a) we show three mass benchmarks from Table 1 and in (b) we show the effect of decreasing the angular resolution of the detector to 60 degrees and of lowering the energy threshold to 40 keVr. (Darker regions indicate where the bands overlap.)

for the whole nucleus. Define the constant $\kappa$ :

$$
\kappa=N_{T} \frac{\rho_{X}}{m_{\chi}} \frac{\sigma_{n} m_{N}}{2 \mu_{n}^{2}} \frac{\left(f_{p} Z+(A-Z) f_{n}\right)^{2}}{f_{n}^{2}} .
$$

Changing variables to $\vec{v}^{\prime}=\vec{v}+\vec{v}_{E}$ gives:

$$
\frac{d R}{d E_{R} d \cos \gamma}=\kappa F^{2}\left(E_{R}\right) \int d^{3} v f(\vec{v}) \delta^{(1)}\left(\vec{v} \cdot \hat{v}_{R}-\vec{v}_{E} \cdot \hat{v}_{R}-v_{\min }\left(E_{R}, \delta\right)\right)
$$

and $F^{2}\left(E_{R}\right)$ is the Helm form factor given in 54]. This formula is discussed in detail (in the context of Radon transforms) in [55]. Thus we can see that at fixed $E_{R}$, the signal peaks where the delta function is nonzero over the largest portion of the phase space, or $\cos \gamma=\hat{v}_{E} \cdot \hat{v}_{R}=-1$. The peak in $E_{R}$ and fixed $\gamma$ is determined by the competition between the form factor (which pushes the signal to lower energies) and the inelasticity (whereby the minimum velocity produces a minimum value of $E_{R}$ ).

Following [22], we use the truncated Maxwell-Boltzmann distribution in the rest of this paper:

$$
f(\vec{v})=\frac{1}{n\left(v_{0}, v_{\text {esc }}\right)} \exp \left(-\frac{\vec{v}^{2}}{v_{0}^{2}}\right) \Theta\left(v_{\text {esc }}-|\vec{v}|\right)
$$

where $n\left(v_{0}, v_{\text {esc }}\right)$ normalizes $\int d^{3} v f$ to 1 . The resulting spectrum is:

$$
\frac{d R}{d E_{R} d \cos \gamma}=\frac{\kappa F^{2}\left(E_{R}\right)}{n\left(v_{0}, v_{e s c}\right)} \pi v_{0}^{2}\left(\exp \left(-\frac{\left(\vec{v}_{E} \cdot \hat{v}_{R}+v_{\min }\left(E_{R}, \delta\right)\right)^{2}}{v_{0}^{2}}\right)-\exp \left(-\frac{v_{\text {esc }}^{2}}{v_{0}^{2}}\right)\right) \Theta\left(v_{\text {esc }}-\left|\vec{v}_{E} \cdot \hat{v}_{R}+v_{\min }\left(E_{R}, \delta\right)\right|\right)(8)
$$

The values we use for the astrophysical parameters are: $v_{0}=220 \mathrm{~km} / \mathrm{s}, v_{E}=225 \mathrm{~km} / \mathrm{s}, v_{\text {esc }}=500-600 \mathrm{~km} / \mathrm{s}$ [56], and $\rho_{\chi}=0.3 \mathrm{GeV} / \mathrm{cm}^{3}$. The normalized rate spectrum of several benchmark models is shown in Fig. 1.

\section{SENSITIVITY}

A robust detection of a directional modulation is possible with surprisingly few events, and does not require use of the rate formulas in the previous section. In fact, a full likelihood analysis based on the correct model is only a factor of $\sim 2$ better than a simple technique, and for a convincing detection, simpler is better. In this section we assume the detection gas has $A=127$ (for iodine; Xe with $A=131$ would be similar) and focus on the energy range $E_{R} \in[50,80] \mathrm{keVr}$.

\section{A. Detectability}

For a model-independent statistic we follow [47, 57 and use the dipole of the recoil direction, $\langle\cos \gamma\rangle$. This is motivated by the fact that the rate should depend only 
on $\cos \gamma$ and $E_{R}$, so the directional part can be expanded in spherical harmonics.

Our detection criterion is a measurement of $\langle\cos \gamma\rangle$ that is $5 \sigma$ relative to the distribution of $\langle\cos \gamma\rangle$ for the same number of randomly distributed events. For a fixed exposure, we generate many random sets of model data (constrained by the DAMA benchmarks in Table 【), and then demand that $90 \%$ of the time the result is $5 \sigma$ from the null hypothesis. The background is modeled as uniform in recoil energy and angle. We assume the detector has an angular resolution of 15 degrees.

In Fig. 2(a) we show the exposures necessary for such conditions, as a function of the background rate, for a few benchmark models. At zero background, roughly 18 events are needed for all benchmark models, on average. Fig. 2(b) shows the effect of decreasing the angular resolution to 60 degrees and lowering the energy threshold of the experiment to $E_{R}=40 \mathrm{keVr}$. Because of the sharp angular profile of the recoil spectrum, a poor angular resolution does not significantly reduce the possibility of a detection. However, achieving an energy threshold of $30-40 \mathrm{keVr}$ dramatically lowers the necessary exposures because the peak of the recoil spectrum occurs at 30-40 keVr and falls off exponentially.

\section{B. Parameter Estimation}

We also perform a likelihood analysis as a measure of sensitivity of the experiment to the parameters of the model, assuming perfect energy and angular resolution. From our analysis in the previous section, we expect this assumption does not affect the results significantly. (See also [48], which shows the sensitivity dependence on angular resolution.) The parameters we consider here are $m_{\chi}, \delta$, and $\sigma_{n}$, which we denote together simply by $p$. Define

$$
\mu(x ; p) \equiv \frac{d R}{d E_{R} d \cos \gamma}(x ; p)+d R_{B G} / 2,
$$

which is the rate $(\mathrm{cpd} / \mathrm{kg} / \mathrm{keVr}$ per $\cos \gamma)$ at a given recoil energy and angle (denoted together by $x$ ) for parameters $p$. We assume the background rate, $d R_{B G}$, in units of $\mathrm{cpd} / \mathrm{keVr} / \mathrm{kg}$, is known.

The likelihood is the probability of parameters $p$ given the events $\left\{x_{i}\right\}$. Given events $\left\{x_{i}\right\}$, bin the events such that in each bin there is only 0 or 1 event and label the bins with one count by $\left\{X_{\alpha}\right\}$ and the empty bins by $\left\{X_{\beta}\right\}$. The expected number of counts in a bin is

$$
E(X ; p)=\mathcal{E} \mu(x ; p) \Delta x
$$

where $\mathcal{E}$ is the exposure. Then the (log) likelihood is

$$
\ln \mathcal{L}_{t o t}(p)=\sum_{\alpha} \ln \left(e^{-E\left(X_{\alpha} ; p\right)} E\left(X_{\alpha} ; p\right)\right)+\sum_{\beta} \ln e^{-E\left(X_{\beta} ; p\right)}
$$

which is the log of the Poisson probability of obtaining 0 or 1 event in each bin. To find the expected average $\ln \mathcal{L}_{\text {tot }}$ for a given exposure $\mathcal{E}$ and true parameters $p_{0}$, we compute

$$
\ln \mathcal{L}_{\text {tot }}(p)=\mathcal{E} \int d x\left(\mu\left(x ; p_{0}\right) \ln \mu(x ; p)-\mu(x ; p)\right)
$$

which is the continuum, noiseless limit of Eq. 11. Since we can only compare differences in log likelihood, in this equation we have subtracted an arbitrary constant in $p$ which takes care of the units in $\ln \mu(x, p)$.

In Figs. 3-9 we show confidence levels of $(68,90,95$, 99, and $99.9 \%$ ) on the WIMP parameters for an exposure of $1000 \mathrm{~kg}$ - day. To obtain the probability, or likelihood, at a point in the $m_{\chi}-\delta$ plane, we either: 1) find the likelihood as a function of $\sigma_{n}$ and maximize with respect to $\sigma_{n}$ or 2) assume $\sigma_{n}$ is exactly known from some other experiment. We can do the same also for points in $m_{\chi}-$ $\sigma_{n}$ plane and $\sigma_{n}-\delta$ plane. The full $\log$ likelihood function lives in the full 3 dimensional parameter space. Here we show possible slices through that space.

For each possible slice, we have shown several variations on the real WIMP parameters or experimental parameters. In the default scenario, we consider the $m_{\chi}=150 \mathrm{GeV}$ benchmark with $E_{t h}=50 \mathrm{keVr}$, a background rate of $d R_{B G}=10^{-3} \mathrm{cpd} / \mathrm{kg} / \mathrm{keVr}$, and $v_{\text {esc }}=$ $500 \mathrm{~km} / \mathrm{s}$. We consider the following independent variations:

- Lower energy threshold $\left(E_{t h} \rightarrow 40 \mathrm{keVr}\right)$

- Higher background $\left(d R_{B G} \rightarrow 10^{-2} \mathrm{cpd} / \mathrm{kg} / \mathrm{keVr}\right)$

- Higher escape velocity $\left(v_{e s c}=600 \mathrm{~km} / \mathrm{s}\right)$

- Lower WIMP mass $\left(m_{\chi} \rightarrow 70 \mathrm{GeV}\right.$ benchmark)

- Higher WIMP mass $\left(m_{\chi} \rightarrow 700 \mathrm{GeV}\right.$ benchmark)

In each case, as $m_{\chi}$ and $v_{\text {esc }}$ vary, $\sigma_{n}$ and $\delta$ are adjusted to agree with benchmark fits to DAMA, using the parameters in Table [1] At masses above $250 \mathrm{GeV}$, there is increasing tension between the DAMA result and other experiments, notably CDMS. This tension is highly dependent on the high velocity tail of the WIMP velocity distribution, and can be alleviated by considering non-Maxwellian velocity distributions, for instance from the Via Lactea simulation [38, 58]. Thus, we consider these points, but it should be emphasized that the non-Maxwellian halos generally tend to lead to a larger signal at DAMA (relative to the other experiments), and thus on a xenon target (because of the similar kinematics), and thus we expect that our use of a Maxwellian distribution is conservative for these points.

At masses much larger than the nucleus mass, the threshold velocity $v_{\min }$ is independent of mass and the spectrum depends on $m_{\chi}$ only through the local WIMP density $\rho_{\chi} / m_{\chi}$. In these regions $m_{\chi}$ and $\sigma_{n}$ are completely degenerate since only the combination $\rho_{\chi} \sigma_{n} / m_{\chi}$ ever appears, as a prefactor determining the overall rate. This can be clearly seen in Fig. 3, which shows confidence intervals in the $m_{\chi}-\sigma_{n}$ plane. Note that because the 
contours never close, we have have imposed the (rather conservative) constraint that $m_{\chi}<100 \mathrm{TeV}$ based on the unitarity bound [59] for a thermal relic.

The effects of the $m_{\chi}-\sigma_{n}$ degeneracy can also be seen in the $m_{\chi}-\delta$ plane, shown in Fig. 5. Here high masses are all equally likely (given a fixed $\delta$ ) because $\sigma_{n}$ can be adjusted accordingly.

In the $\delta-\sigma_{n}$ plane, Fig. 7 there is a sharp discontinuity since low masses are favored at smaller $\sigma_{n}$ and very high masses are favored at high $\sigma_{n}$. This is because at low scattering cross section, in order to boost the rates such that it matches the observed number of events, one can lower $\delta$ or adjust the mass to optimize the number of rates. (The scattering rate is maximized when the mass of the WIMP $\sim$ the mass of the nuclei.) However, at high scattering cross section, one can increase $\delta$ but only increase the mass to very high masses to reduce the rates. Though lowering the mass drastically also decreases the rate, the angular shape at very low masses is very distinct (see Fig. 1) and thus unfavored. The cutoff in Fig. 7 at high $\sigma_{n}$ is a result of the unitarity bound on the mass.

These effects can make it difficult to constrain the WIMP mass at low exposures; however, it is easier to constrain the ratio $m_{\chi} / \sigma_{n}$, which we have shown in Fig. 9 ,

Finally, we note that in these figures we have assumed the earth velocity is unmodulated. For the benchmark where $m_{\chi}=70 \mathrm{GeV}$, our worst-case scenario, the effects of the annual modulation in velocity can improve the confidence levels significantly if the experiment is done during the summer.

The disadvantage of the likelihood analysis is its model dependence. We used the truncated Maxwell-Boltzmann profile, whereas in reality it is likely there is more structure in the dark matter profile. However we expect the results to roughly be the same for many more complicated velocity distributions, and in fact can improve for inelastic dark matter, as mentioned above. Furthermore, because of the velocity threshold due to $\delta$, the inelastic scenario is not very sensitive to streams because most streams are below the threshold velocity. Anisotropies in the halo profile do not significantly affect the results here. To see the effect of using less simplistic halo models on the elastic scattering spectrum and sensitivity, see [60] and [61].

\section{CONCLUSIONS}

Motivated by the finding 22] that inelastic dark matter (iDM) is compatible with both the DAMA annual modulation signal at $22-66 \mathrm{keVr}$ and limits from other experiments at lower energies, we have investigated prospects for directional detection in the context of the iDM model. We are encouraged by the fact that ZEPLIN-III has also detected a number of events in the $40-80 \mathrm{keVr}$ range [62]. This has not been claimed as evidence of WIMP scattering, but makes it impossible to rule out
iDM with such data. In the near future, LUX [65] and XENON100 63] will have greatly improved sensitivity and lower backgrounds, and will provide a sharp test of the iDM/DAMA scenario. If these experiments also detect an excess of events above background in the appropriate energy range, a major effort in directional detection will be justified.

Directional detection with a gaseous detector containing a heavy gas (e.g. Xe) may not require the huge exposure times implied by the elastic scattering limits. For a threshold energy of $E_{t h}=50 \mathrm{keVr}$, we find that exposures of order $\sim 1000 \mathrm{~kg} \cdot$ day in a directional experiment can convincingly refute or support the claims of DAMA in the context of the inelastic dark matter model. At zero background, roughly 18 events are needed for a clear detection of WIMP scattering. Even with larger backgrounds, the required exposure is a few hundred $\mathrm{kg} \cdot$ day, over most of the iDM parameter space that can explain both DAMA and other direct detection experiments. With roughly $1000 \mathrm{~kg}$. day, it is possible to obtain a measurement of $\delta>0$ at high significance and also the parameter $m_{\chi} / \sigma_{n}$ to within an order of magnitude.

Furthermore, if it is possible to roughly determine one of the WIMP parameters, for example $\delta \sim 120 \mathrm{keV}$, via another experiment, the mass and nucleon scattering cross section are highly constrained with an exposure of a few hundred $\mathrm{kg}$ - day because of the distinctive shape of the energy-angle recoil spectrum.

Significantly lower exposures are needed if the threshold energy is decreased. As discussed in Section III because of the uncertainties in the nuclear recoil energies of the DAMA signal, it is crucial to reduce the threshold energy as much as possible. For low masses, the recoil spectrum is sharply distributed in energy and angle. However, typical recoil energies are smaller. Thus with an energy threshold of $E_{t h}=50 \mathrm{keVr}$ most of the events for $m_{\chi}=70 \mathrm{GeV}$ are not seen. With an energy threshold of $100 \mathrm{keVr}$ and $m_{\chi}=70 \mathrm{GeV}$, none of the WIMP recoils can be seen. Though the required volume increases and angular resolution decreases when $E_{t h}$ is lowered, we found that a poor angular resolution $\left(\sim 60^{\circ}\right)$ does not significantly affect the results, assuming that $3 \mathrm{D}$ reconstruction of the track and determining the sense is still possible.

We acknowledge helpful discussions about directional detection with Peter Fisher, Jocelyn Monroe, and Gabriella Sciolla. Rick Gaitskell, Dan McKinsey, and Peter Sorensen provided helpful advice and much-needed skepticism. DPF is partially supported by NASA LTSA grant NAG5-12972. TL is supported by an NSF Graduate Fellowship. This work was partially supported by the Director, Office of Science, of the U.S. Department of Energy under Contract No. DE-AC02-05CH11231. NW is supported by NSF CAREER grant PHY-0449818 and DOE OJI grant \#DE-FG02-06E R41417. 

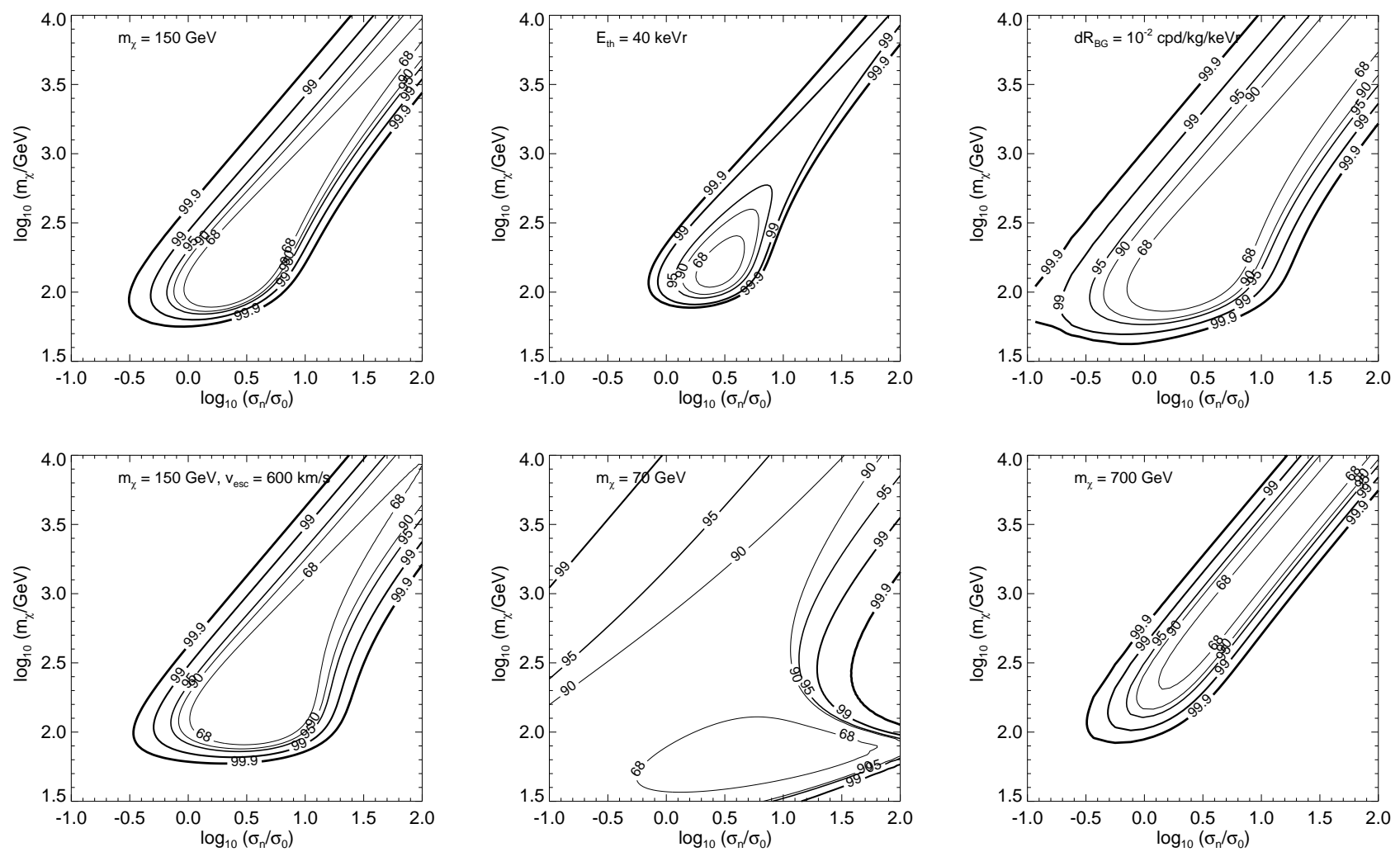

FIG. 3: Confidence levels for determining $m_{\chi}$ and $\sigma_{n}$, where $\delta$ is unknown, with an exposure of $1000 \mathrm{~kg} \cdot$ day. $\sigma_{0}=10^{-40} \mathrm{~cm}^{2}$.
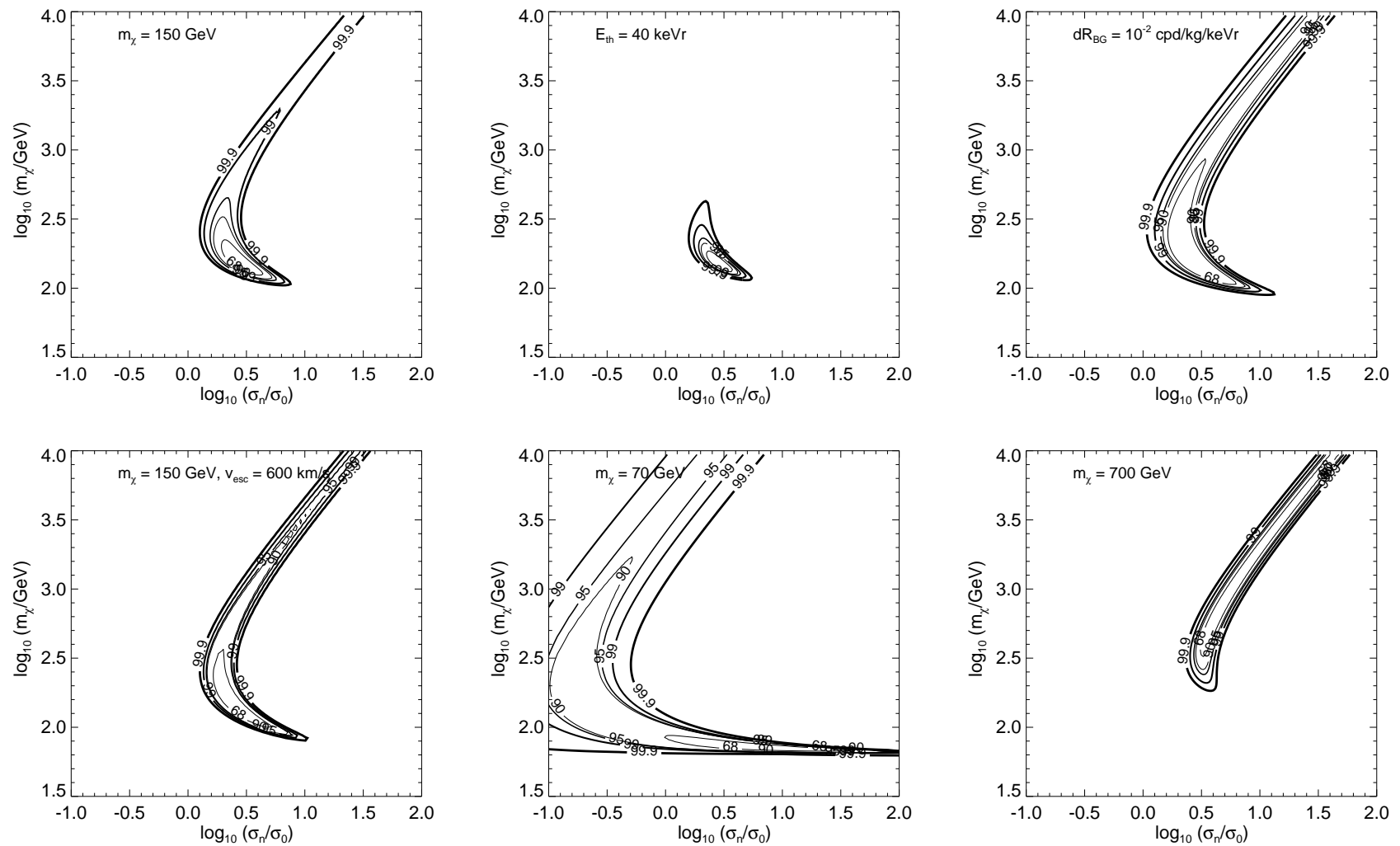

FIG. 4: Confidence levels for determining $m_{\chi}$ and $\sigma_{n}$, where $\delta$ is known with an exposure of $1000 \mathrm{~kg} \cdot$ day. $\sigma_{0}=10^{-40} \mathrm{~cm}^{2}$. 

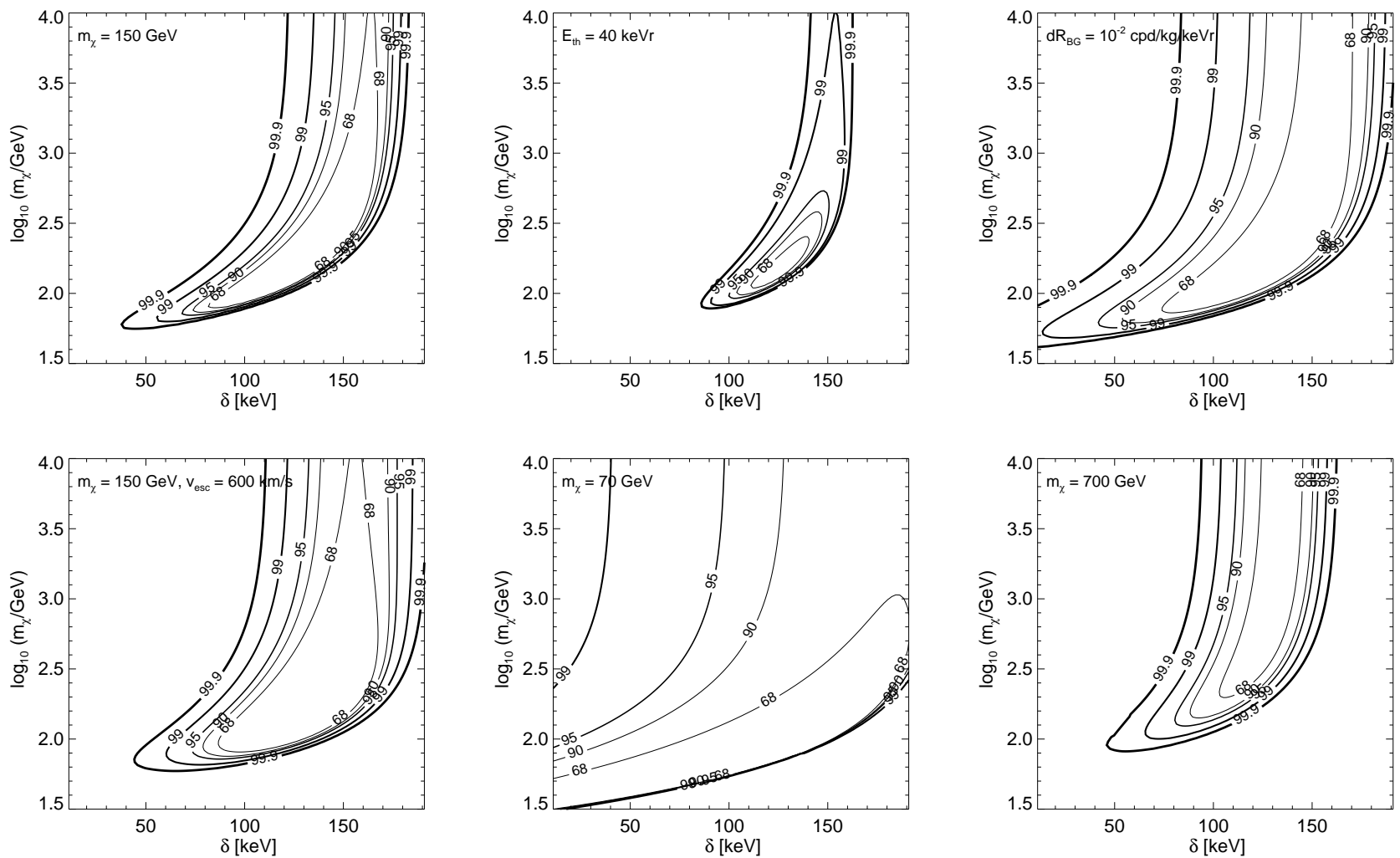

FIG. 5: Confidence levels for determining $m_{\chi}$ and $\delta$, where $\sigma_{n}$ is unknown, with an exposure of $1000 \mathrm{~kg}$. day.
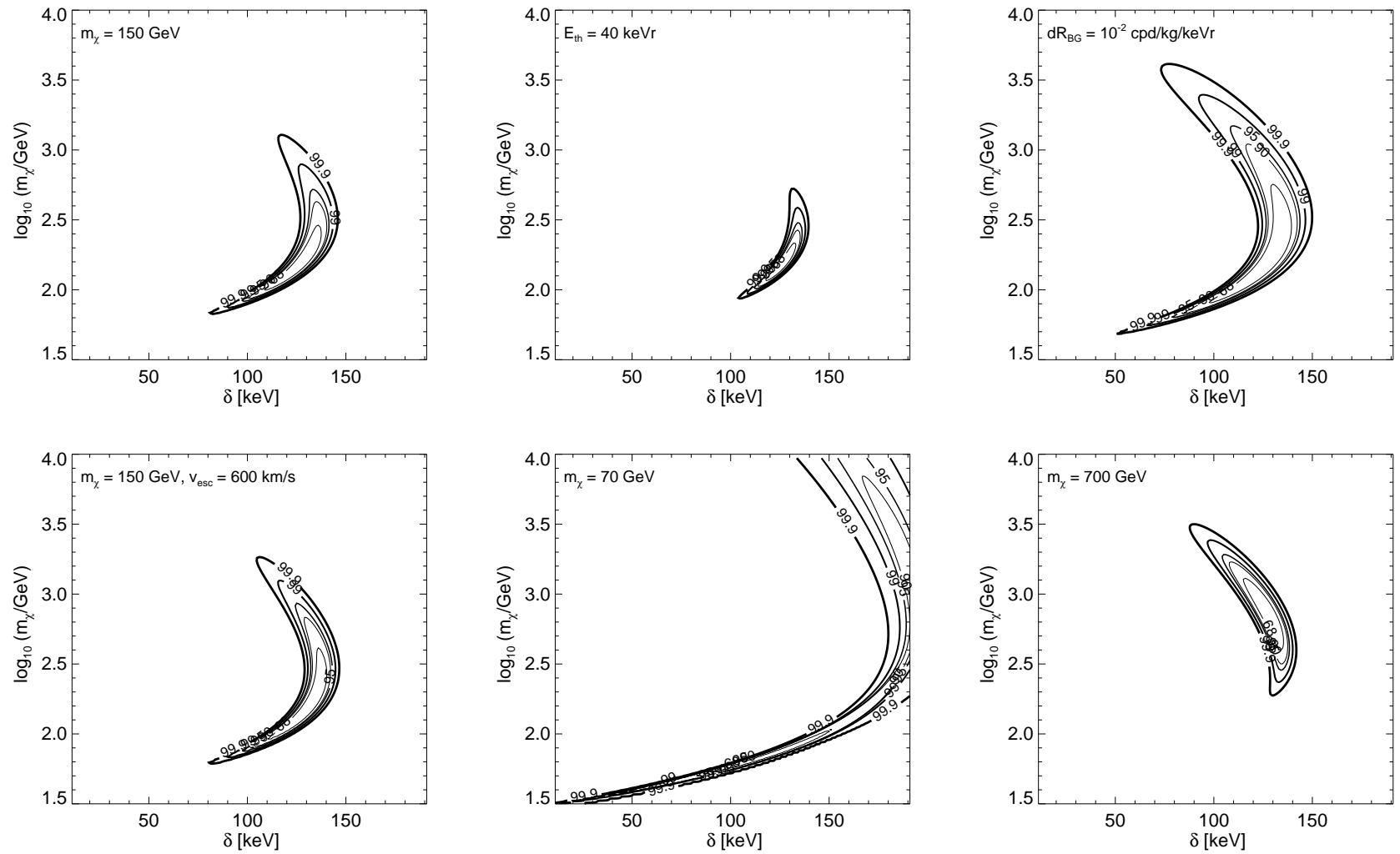

FIG. 6: Confidence levels for determining $m_{\chi}$ and $\delta$, where $\sigma_{n}$ is known, with an exposure of $1000 \mathrm{~kg} \cdot$ day. 

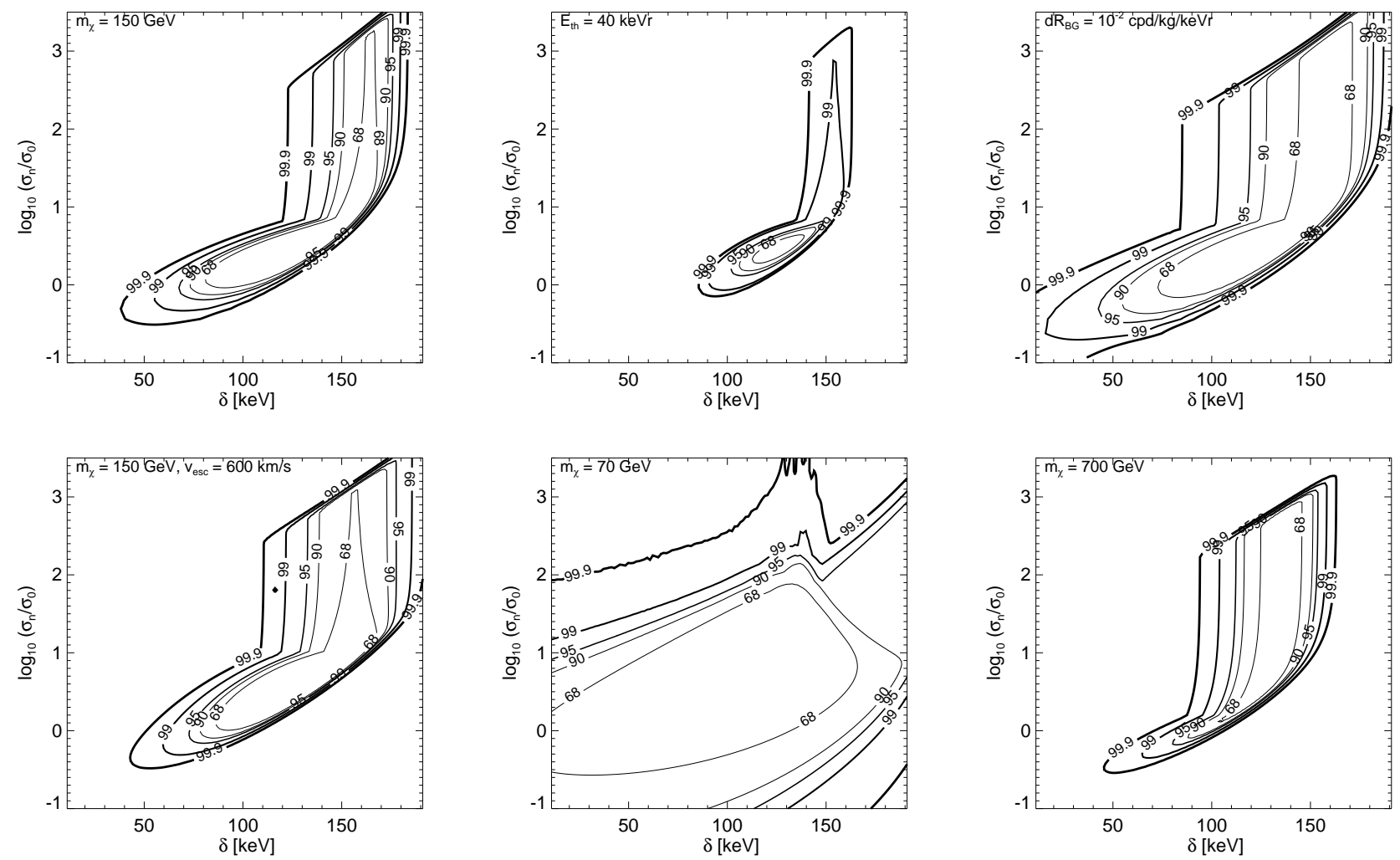

FIG. 7: Confidence levels for determining $\delta$ and $\sigma$, where $m_{\chi}$ is unknown, with an exposure of $1000 \mathrm{~kg} \cdot$ day. $\sigma_{0}=10^{-40} \mathrm{~cm}^{2}$.
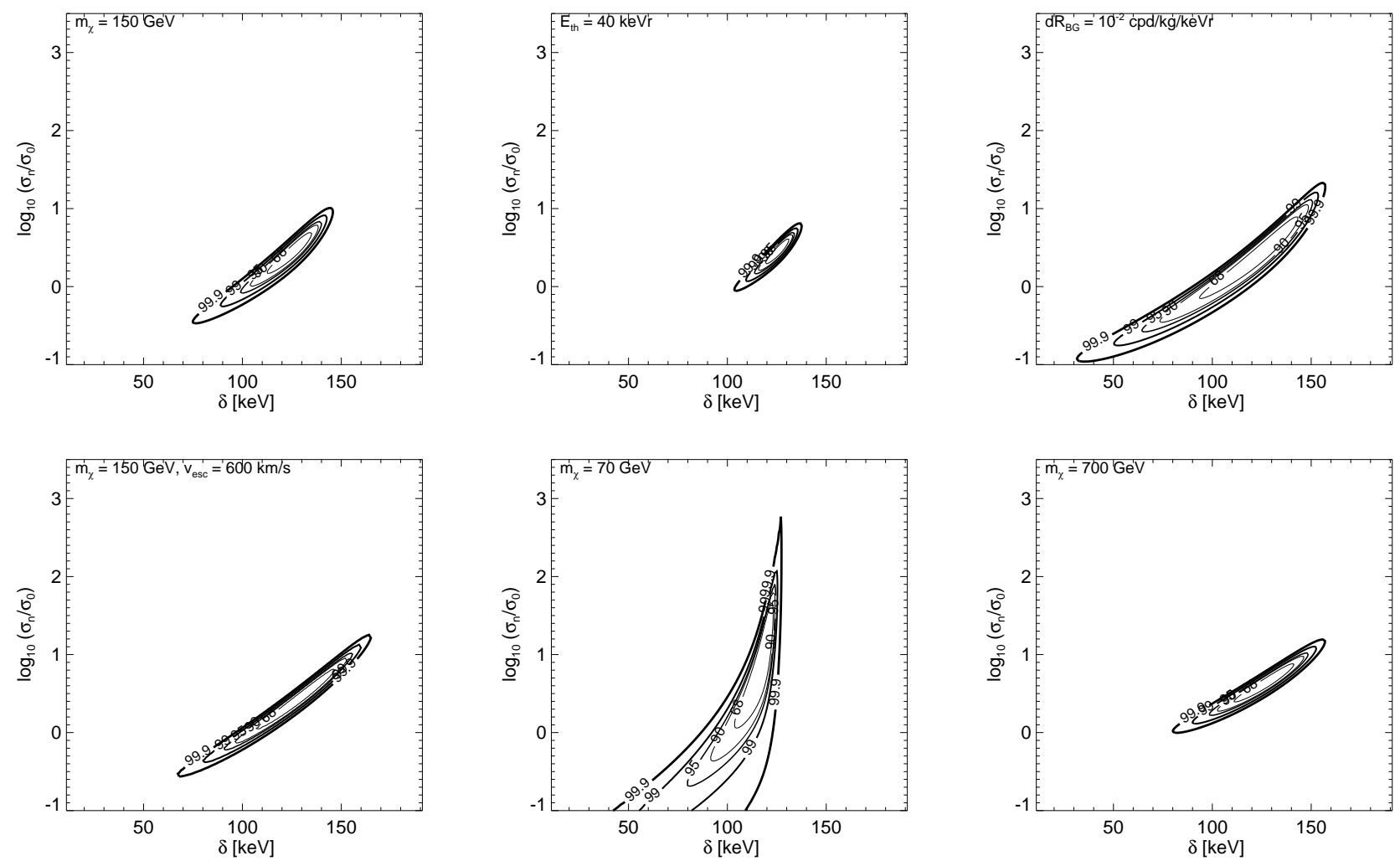

FIG. 8: Confidence levels for determining $\delta$ and $\sigma_{n}$, where $m_{\chi}$ is $k n o w n$, with an exposure of $1000 \mathrm{~kg} \cdot \mathrm{day} \cdot \sigma_{0}=10^{-40} \mathrm{~cm}^{2}$. 

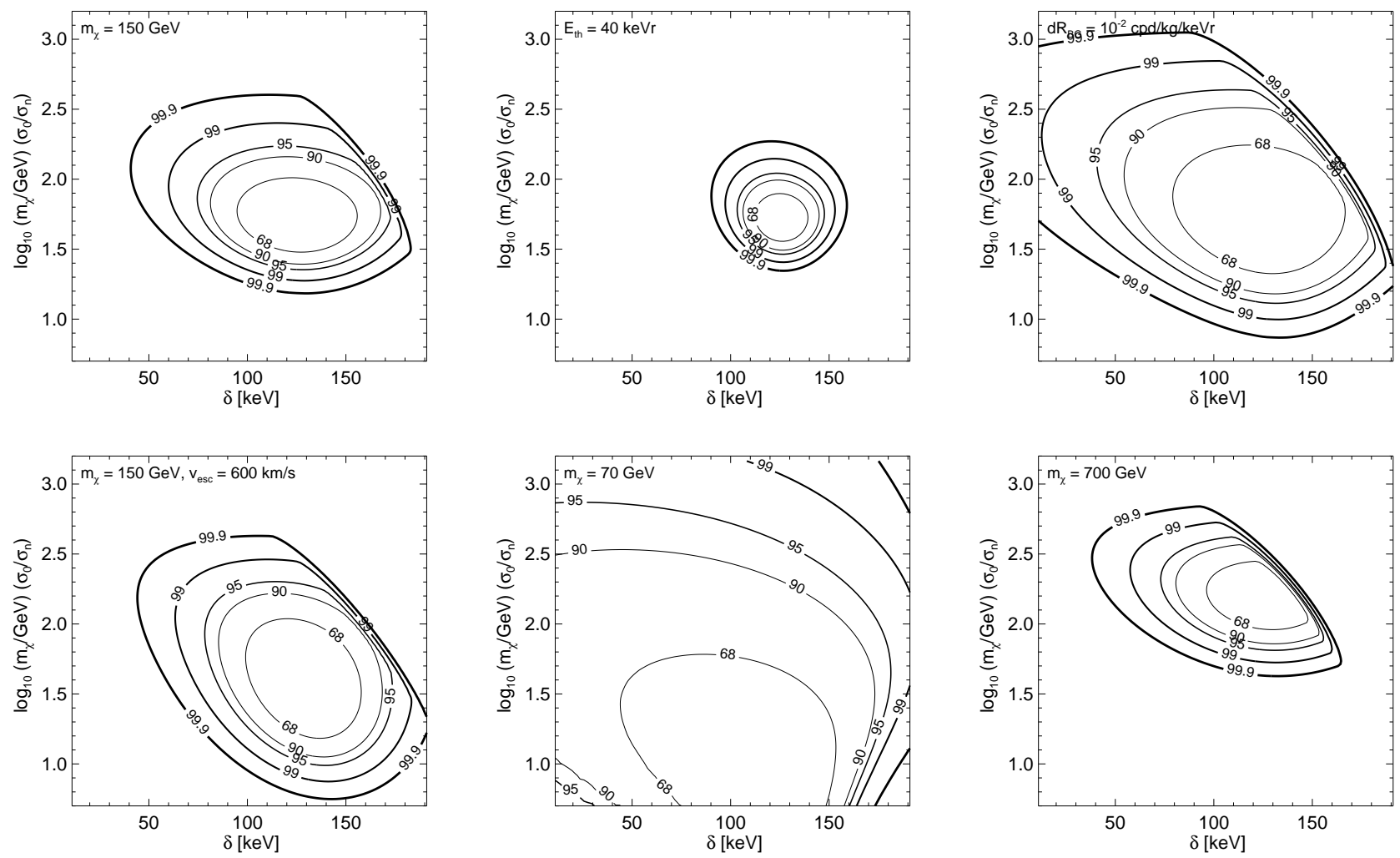

FIG. 9: Confidence levels for determining $\delta$ and $m_{\chi} / \sigma_{n}$, where $m_{\chi}$ is unknown, with an exposure of $1000 \mathrm{~kg} \cdot \mathrm{day}$, taking $\sigma_{0}=10^{-40} \mathrm{~cm}^{2}$. Over most of the parameter space, some value of $m_{\chi}$ (and therefore $\sigma_{n}$ ) can be found to produce enough events for the given $\delta$. However, in the case of large $\delta$ and large $m_{\chi} / \sigma_{n}$, no solution is possible in some cases.

[1] R. Bernabei et al. (DAMA), Phys. Lett. B480, 23 (2000).

[2] R. J. Gaitskell, Ann. Rev. Nucl. Part. Sci. 54, 315 (2004).

[3] J. Angle et al. (XENON10), Phys. Rev. Lett. 100, 021303 (2008), 0706.0039.

[4] J. Angle et al., Phys. Rev. Lett. 101, 091301 (2008), 0805.2939.

[5] Z. Ahmed et al. (CDMS), Phys. Rev. Lett. 102, 011301 (2009), 0802.3530.

[6] R. Bernabei et al. (DAMA), Eur. Phys. J. C56, 333 (2008), 0804.2741.

[7] R. Bernabei et al., Phys. Rev. D77, 023506 (2008), 0712.0562.

[8] P. Ullio, M. Kamionkowski, and P. Vogel, JHEP 07, 044 (2001), hep-ph/0010036.

[9] P. Belli, R. Cerulli, N. Fornengo, and S. Scopel, Phys. Rev. D66, 043503 (2002), hep-ph/0203242.

[10] C. Savage, P. Gondolo, and K. Freese, Phys. Rev. D70, 123513 (2004), astro-ph/0408346.

[11] A. Bottino, F. Donato, N. Fornengo, and S. Scopel, Phys. Rev. D69, 037302 (2004), hep-ph/0307303.

[12] P. Gondolo and G. Gelmini, Phys. Rev. D71, 123520 (2005), hep-ph/0504010.

[13] D. R. Smith and N. Weiner, Phys. Rev. D64, 043502 (2001), hep-ph/0101138.

[14] E. Behnke et al. (COUPP), Science 319, 933 (2008), 0804.2886.

[15] C. Savage, G. Gelmini, P. Gondolo, and K. Freese, JCAP 0904, 010 (2009), 0808.3607.

[16] R. Bernabei et al., Eur. Phys. J. C53, 205 (2008), 0710.0288.

[17] A. Bottino, F. Donato, N. Fornengo, and S. Scopel, Phys. Rev. D77, 015002 (2008), 0710.0553.

[18] F. Petriello and K. M. Zurek, JHEP 09, 047 (2008), 0806.3989.

[19] S. Chang, A. Pierce, and N. Weiner (2008), 0808.0196.

[20] M. Fairbairn and T. Schwetz, JCAP 0901, 037 (2009), 0808.0704.

[21] D. Tucker-Smith and N. Weiner, Phys. Rev. D72, 063509 (2005), hep-ph/0402065.

[22] S. Chang, G. D. Kribs, D. Tucker-Smith, and N. Weiner (2008), 0807.2250.

[23] N. Arkani-Hamed and N. Weiner (2008), 0810.0714.

[24] Y. Cui, D. E. Morrissey, D. Poland, and L. Randall (2009), 0901.0557.

[25] D. S. M. Alves, S. R. Behbahani, P. Schuster, and J. G. Wacker (2009), 0903.3945. 
[26] N. Arkani-Hamed, D. P. Finkbeiner, T. Slatyer, and N. Weiner (2008), 0810.0713.

[27] M. Pospelov and A. Ritz (2008), 0810.1502.

[28] E. J. Chun and J.-C. Park, JCAP 0902, 026 (2009), 0812.0308.

[29] M. Baumgart, C. Cheung, J. T. Ruderman, L.-T. Wang, and I. Yavin, JHEP 04, 014 (2009), 0901.0283.

[30] C. Cheung, J. T. Ruderman, L.-T. Wang, and I. Yavin, Phys. Rev. D80, 035008 (2009), 0902.3246.

[31] A. Katz and R. Sundrum, JHEP 06, 003 (2009), 0902.3271.

[32] D. P. Finkbeiner, T. R. Slatyer, N. Weiner, and I. Yavin, JCAP 0909, 037 (2009), 0903.1037.

[33] B. Batell, M. Pospelov, and A. Ritz, Phys. Rev. D79, 115019 (2009), 0903.3396.

[34] D. E. Morrissey, D. Poland, and K. M. Zurek, JHEP 07, 050 (2009), 0904.2567.

[35] F. Chen, J. M. Cline, and A. R. Frey (2009), 0907.4746.

[36] D. E. Kaplan, G. Z. Krnjaic, K. R. Rehermann, and C. M. Wells (2009), 0909.0753.

[37] D. N. Spergel, Phys. Rev. D37, 1353 (1988).

[38] J. March-Russell, C. McCabe, and M. McCullough (2008), 0812.1931.

[39] R. Bernabei et al. (DAMA), Nucl. Instrum. Meth. A592, 297 (2008), 0804.2738.

[40] G. Sciolla et al. (2009), 0903.3895.

[41] K. Miuchi et al., Phys. Lett. B654, 58 (2007), 0708.2579.

[42] S. Burgos et al., Nucl. Instrum. Meth. A600, 417 (2009), 0807.3969.

[43] D. Santos, O. Guillaudin, T. Lamy, F. Mayet, and E. Moulin, J. Phys. Conf. Ser. 65, 012012 (2007), astro-ph/0703310.

[44] S. Ahlen et al. (2009), 0911.0323.

[45] G. Sciolla (2008), 0811.2764.

[46] G. Sciolla and C. J. Martoff (2009), 0905.3675.

[47] B. Morgan, A. M. Green, and N. J. C. Spooner, Phys. Rev. D71, 103507 (2005), astro-ph/0408047.

[48] C. J. Copi, L. M. Krauss, D. Simmons-Duffin, and S. R. Stroiney, Phys. Rev. D75, 023514 (2007), astro-ph/0508649.

[49] A. M. Green and B. Morgan, Phys. Rev. D77, 027303 (2008), 0711.2234.

[50] D. Dujmic et al., Astropart. Phys. 30, 58 (2008), 0804.4827.

[51] D. P. Snowden-Ifft, C. J. Martoff, and J. M. Burwell, Phys. Rev. D61, 101301 (2000), astro-ph/9904064.

[52] S. Burgos et al., Astropart. Phys. 28, 409 (2007), 0707.1488.

[53] T. Tanimori et al., Phys. Lett. B578, 241 (2004), astro-ph/0310638.

[54] J. D. Lewin and P. F. Smith, Astropart. Phys. 6, 87 (1996).

[55] P. Gondolo, Phys. Rev. D66, 103513 (2002), hep-ph/0209110.

[56] M. C. Smith et al., Mon. Not. Roy. Astron. Soc. 379, 755 (2007), astro-ph/0611671.

[57] A. M. Green and B. Morgan, Astropart. Phys. 27, 142 (2007), astro-ph/0609115.

[58] M. Kuhlen and N. Weiner (in progress) (2009).

[59] K. Griest and M. Kamionkowski, Phys. Rev. Lett. 64, 615 (1990).

[60] M. S. Alenazi and P. Gondolo, Phys. Rev. D77, 043532 (2008), 0712.0053.

[61] C. J. Copi and L. M. Krauss, Phys. Rev. D63, 043507 (2001), astro-ph/0009467.

[62] V. N. Lebedenko et al. (2008), 0812.1150.

[63] E. Aprile and L. Baudis (XENON100) (2009), 0902.4253.

[64] The term experimentum crucis was first used by Isaac Newton in a 1672 letter about his Theory of Light and Colors.

[65] http://lux.brown.edu 